\title{
On the relation between the enactive and the sensorimotor approach to perception
}

\author{
Dario Taraborelli \\ Department of Psychology \\ University College London \\ Gower Street \\ London WC1E 6BT \\ United Kingdom
}

\author{
Matteo Mossio* \\ IHPST \\ CNRS/Université Paris I \\ 13, Rue du Four \\ 75006 Paris \\ France
}

In Mossio \& Taraborelli (2008) we challenged the assumption according to which the ecological and sensorimotor approaches are mere conceptual variations on the same enactive theme. We showed, on the contrary, that they endorse substantially different notions of an "action-dependent perceptual invariant" and we submitted that this distinction has interesting theoretical and empirical implications.

This dissimilarity between ecological and sensorimotor theories stems, in our view, from a more fundamental divergence on the nature of perceptual information. Since Gibson's work, the ecological approach has adopted a fundamentally realist stance, according to which this information is "picked up", "revealed", "encountered" and "exploited" by the organism (as a range of affordances) but not "constituted" by the activity of the organism. In contrast, sensorimotor approaches take an explicit interactivist position, which sees the perceiver's motor activity as a determinant of perceptual information itself.

Pascal \& O'Regan (2008) suggest that our analysis of the relationship between ecological, sensorimotor and enactive theories would benefit from a further distinction. The original enactive approach (Varela et al. 1991; Maturana, 2002; Thompson, 2007), they argue, should not be lumped together with contemporary sensorimotor approaches to perception: whereas sensorimotor theories endorse an externalist view of perception, according to which "perception can only be understood as a form of interaction of the organism with the environment", the original enactive approach by Varela and collaborators would have strong "idealist underpinnings".

To address this criticism, we should first clarify that the enactive approach can be regarded as a general theoretical framework on the nature of biological organisms that spawned a variety of strains of research, in particular:

1. an account of how biological autonomous systems constitute themselves across their history of interaction with the environment;

2. a hypothesis on perception as built upon structural couplings between sensory patterns and motor activity;

3. a critical appraisal of the functionalist approach to the study of phenomenology.

\footnotetext{
* Matteo Mossio's work was partly supported by a postdoctoral fellowship from the Centre National de la Recherche Scientifique.
} 
Pascal \& O'Regan submit that the idealist underpinning of Varela's position can be traced back to his philosophical commitment to an anti-functionalist view of phenomenology, which, in particular, rejects the way in which the problem of consciousness and subjectivity has been framed in mainstream philosophy of mind and cognitive science (3.). However, if we leave aside the phenomenological part of Varela's work, and focus instead on his view on the nature of perception, then the allegation of "idealism" relies on a fundamental misinterpretation of the enactive approach. ${ }^{1}$

There are indeed several valid reasons to distinguish the sensorimotor and enactive approaches. We submit, however, that with respect to their view of perception (2.), enactive and sensorimotor theories are fully compatible, insofar as they endorse a substantially identical idea of perceptual information. In this sense, the opposition between externalist sensorimotor theories and idealist enactive theories suggested by Pascal \& O'Regan does not seem to point to any relevant conceptual difference between the two approaches.

As Varela puts it:

[t]he enactive approach underscores the importance of two interrelated points: 1) perception consists of perceptually guided action and 2) cognitive structures emerge from the recurrent sensorimotor patterns that enable action to be perceptually guided. (Varela et al., 1991, p. 173)

To better understand this characterization, it is worth recalling that one of the tenets of the enactive approach to cognition and perception is the notion of structural coupling:

"Structural coupling" refers to the history of recurrent interactions between two or more systems that leads to a structural congruence between them. (Thompson, 2007, p. 45. See also Maturana \& Varela, 1987)

The concept of structural coupling refers to the fact that living systems maintain their identity in a permanent interaction with the external environment so as to preserve their internal organization. Perception and cognition are specific dimensions of the activity in virtue of which biological systems control their relation to the physical world and their own identity. As Varela concludes:

“[w]e found a world enacted by our history of structural coupling" (Varela et al., p. 217).

Far from adopting an idealist perspective on perception, the enactive approach explicitly endorses what Pascal \& O'Regan call an externalist stance, given the essential role of sensorimotor interactions with the environment for the self-maintenance of the system. This assumption is substantially equivalent to the position found in O'Regan and Noë (2001) and Noë (2004) when they stress that perception consists in the knowledge of contingencies that link actions with sensory transformations. In conclusion, we believe that the enactive and the sensorimotor approach cannot be theoretically distinguished with respect to their interpretation of the nature of perception or their hypotheses on the relation between the perceiving agent and the environment (2.).

If we consider enaction as a theory of self-organizing autonomous systems (1.), we agree with Pascal \& O'Regan that "the use of the word enactive by many sensorimotor theorists leads to confusion", but we disagree on why this is the case. The fundamental difference between the enactive and the sensorimotor approaches is that whereas the enactive theory of cognition and perception is embedded in a general theory of biological organization, the sensorimotor view of

\footnotetext{
${ }^{1}$ Varela et al. (1991) explicitely reject any idealist interpretation of perception and cognition by noting that enactivism takes a middle path between "the Scylla of cognition as the recovery of a pregiven outer world (realism) and the Charybdis of cognition as the projection of a pregiven inner world (idealism)" (p.172).
} 
perception might, in principle, be formulated without any commitment to specific biological constraints on the observer.

The enactive approach puts a variety of physical and structural constraints on the kind of systems that can possibly develop cognitive and perceptual capacities. Systems capable of perception and cognition, according to the original enactive view, need to be living systems (or at least possess all the relevant properties that characterize biological organisms) (Maturana \& Varela, 1980). Sensorimotor theories, in contrast, do not make any particular distinction on the kind of systems that may display perceptual abilities in virtue of sensorimotor invariants. From a sensorimotor perspective, the capacity to detect and use sensorimotor invariants does not seem to originate from, or be restricted to, biological organization. The same sensorimotordependent capacities could, in principle, be implemented in a variety of artificial systems (Philipona et al., 2003), an idea on which enactive theories would be extremely cautious, at the very least.

To sum up, we maintain that-leaving aside phenomenological considerations that were not within the scope of our analysis in Mossio \& Taraborelli (2008)-enactive and sensorimotor approaches are fully compatible with respect to their view of perception and can then be contrasted as a whole with ecological approaches. They can however be distinguished from each other with respect to their explanatory scope and on the constraints they put on systems capable of sensorimotor-based perceptual abilities.

\section{References}

1. Maturana, H.R., Varela, F (1980). Autopoiesis and Cognition. Dordrecht: Kluwer.

2. Maturana, H.R., Varela, F. (1987). The tree of knowledge. Boston: New Science Library.

3. Mossio, M., Taraborelli, D. (2008). Action-dependent perceptual invariants: From ecological to sensorimotor approaches, Consciousness and Cognition, in press.

4. Noë, A. (2004). Action in perception, Cambridge, MA: MIT Press.

5. O'Regan, J.K., Noë, A. (2001). A sensorimotor account on vision and visual consciousness, Behavioral and Brain Sciences, 24(5), 939-1011.

6. Pascal, F.P. \& O'Regan, J.K. (2008). Commentary On Mossio and Taraborelli: Is the enactive approach really sensorimotor?, Consciousness and Cognition, in press.

7. Philipona, D., O'Regan, J.K., and Nadal, J.-P. (2003) Is there something out there? Inferring space from sensorimotor dependencies. Neural Computation 15, 9.

8. Thompson, E. (2007). Mind in Life: Biology, Phenomenology, and the Sciences of Mind. Cambridge, MA: Belknap Press.

9. Varela F., Thompson E., \& Rosch E. (1991). The Embodied Mind: Cognitive Science and Human Experience. Cambridge, MA: MIT Press. 